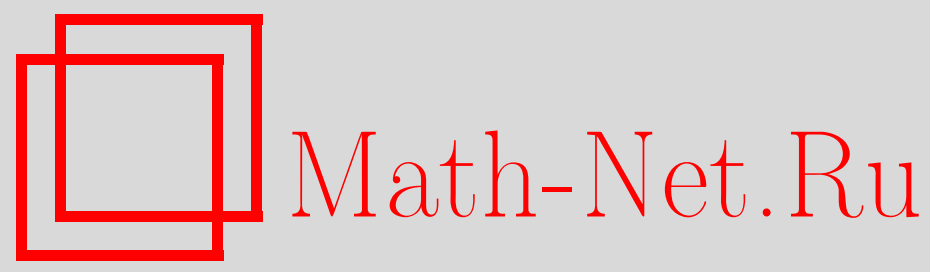

Д. И. Пионтковский, О свободных произведениях в многообразиях ассоциативных алгебр, Матем. заметки, 1999, том 65, выпуск 5, 693-702

DOI: https://doi.org/10.4213/mzm1101

Использование Общероссийского математического портала Math-Net.Ru подразумевает, что вы прочитали и согласны с пользовательским соглашением http://www.mathnet.ru/rus/agreement

Параметры загрузки:

IP : 3.93.64.190

26 апреля 2023 г., 13:29:51

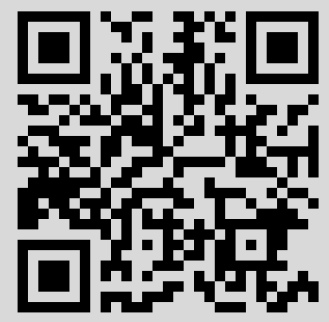




\section{О СВОБОДНЫХ ПРОИЗВЕДЕНИЯХ \\ В МНОГООБРАЗИЯХ АССОЦИАТИВНЫХ АЛГЕБР}

\section{Д. И. Пионтковский}

Рассматриваются многообразия ассоциативных алгебр над полем нулевой характеристики. Недавно А. Я. Белов доказал, что во всяком таком многообразии ряд Гильберта относительно свободной алгебры конечного ранга рационален. Между тем, в многообразии алгебр с нулевым умножением, многообразиях коммутативных алгебр и всех ассоциативных алгебр справедливо и более сильное утверждение. А именно, для этих многообразий хорошо известны формулы, рационально выражающие ряд Гильберта алгебры свободного произведения алгебр через ряды Гильберта сомножителей. В статье приводится система контрпримеров, показьвающая, что в любом другом многообразии аналогичной формулы не существует, даже если один из двух сомножителей - свободная алгебра. Однако, если ограничиться классом градуированньг PI-алгебр, порожденных своими компонентами первой степени, то существует бесконечно много многообразий, для каждого из которых аналогичная формула имеет место.

Библиограффия: 3 названия.

Введение. Пусть $W$ - многообразие ассоциативных алгебр над полем $k$ характеристики 0. Связно градуированными будем назьвать алгебры без единицы, $\mathbb{Z}$-градуированные, с нулевыми неположительными компонентами и конечномерные в каждой компоненте. Ассоциированную с $W$ категорию связно градуированных PI-алгебр будем обозначать той же буквой $W$.

Если элементам не более чем счетного множества $X=\left\{x_{1}, x_{2}, \ldots\right\}$ приписаны положительные степени $d_{1}, d_{2}, \ldots$, то через $F^{W}(X)$ обозначим свободную алгебру многообразия $W$ с множеством порождающих $X$. Для $A, B \in \mathrm{Ob} W$ через $A \stackrel{W}{*} B$ обозначаем свободное произведение в многообразии $W$.

Недавно А.Я. Белов доказал, что во всяком таком многообразии $W$ ряд Гильберта алгебры $F^{W}(X)$ для конечного $X$ рационален [1]. Между тем, в многообразии алгебр с нулевым умножением Vect (мы отождествляем такие алгебры с изоморфными им градуированными векторными пространствами), многообразиях коммутативных алгебр Com и всех ассоциативных алгебр Ass справедливо и более сильное утверждение. А именно, для этих многообразий известны формулы, рационально выражающие ряд Гильберта алгебры $A \stackrel{W}{*} B$ через ряды Гильберта алгебр $A$ и $B:$ точнее, если $a(t)=A(t)+1$,

Работа выполнена при поддержке Российского фонда фундаментальных исследований, грант № 96-01-00149. 
$b(t)=B(t)+1, c(t)=(A \stackrel{W}{*} B)(t)+1$, то для лежащих в соответствующих многообразиях алгебр $A$ и $B$ имеем

$$
\begin{array}{rlrl}
A \stackrel{\text { Vect }}{*} B=A \oplus B, & c(t) & =a(t)+b(t)-1, \\
A \stackrel{\text { Com }}{*} B=A \otimes B, & c(t) & =a(t) \cdot b(t), \\
A \stackrel{\text { Ass }}{*} B=A \sqcup B, & \frac{1}{c(t)}=\frac{1}{a(t)}+\frac{1}{b(t)}-1 .
\end{array}
$$

В связи с этим возникают следующие вопросы.

Вопрос 1. Существуют ли многообразия, в которых ряд Гильберта свободного произведения алгебр определяется рядами Гильберта сомножителей?

Вопрос 2. Существуют ли многообразия, в которых ряд Гильберта свободного произведения алгебр определяется рядами Гильберта сомножителей, если один из сомножителей - свободная алгебра?

В этой заметке показьвается, что ответ на оба вопроса для всякого многообразия $W$, кроме трех перечисленных, отрицателен. Тем самым, на этом пути упомянутый результат Белова является наиболее сильным.

Работа проходила следующим образом. Сначала соответствующие контрпримеры были найдены с помощью компьютера (в частности, для вычисления рядов Гильберта я использовал программу GRAAL, написанную в г. Ульяновске А. Кондратьевым). При этом удалось найти систему контрпримеров, которыепочти не зависят от тождеств четвертой и более высоких степеней: теперь перебор стал сравнительно небольшим, и стало возможным получить представленное здесь "ручное" доказательство.

В п. 1 приводятся некоторые технические утверждения. Самым важным из них является классификация многообразий, с помощью которой далее строятся примеры. Это происходит в п. 2. В п. 3 рассматривается вопрос об алгебрах, порожденных своими элементами первой степени: оказывается, при таком сужении задачи удается найти примеры многообразий, в которых ряд Гильберта свободного произведения алгебр можно вычислить, зная только ряды Гильберта сомножителей. Тем не менее, для произвольного многообразия $W$ даже в таком классе алгебр ответы на оба поставленных вопроса отрицательны.

1. Классификация многообразий и технические леммы. В этом пункте собрано несколько несложных технических утверждений. Вероятно, они известны, однако, за исключением леммы 2 найти их в литературе не удалось.

Будем обозначать через $F_{n}^{W}$ или $F_{n}$ свободную алгебру многообразия $W$ ранга $n$ со стандартной градуировкой (степени порождающих равны 1). Для градуированной алгебры $A$ из $W$ через $\bar{A}$ обозначим изоморфное ей градуированное векторное пространство: другими словами, $::$ : $\Rightarrow$ Vect - функтор забывания мультипликативной структуры. Для элемента $a \in A$ через $\bar{a}$ будем обозначать его образ в $\bar{A}$. Для $W \neq$ Ass через $m$ обозначим наименьшую из степеней тождеств, вьполняющихся в $W$.

ЛЕмма 1. а) Если полилинейное тождество третьей степени $f \equiv 0$ не имеет следствий - нетривиальных тохдеств третьей степени от двух переменных, 
то оно пропорционально стандартному тохсдеству

$$
\operatorname{St}_{3}\left(x_{1}, x_{2}, x_{3}\right)=\sum_{\sigma \in S_{3}}(-1)^{\sigma} x_{\sigma 1} x_{\sigma 2} x_{\sigma 3} \equiv 0 .
$$

б) Если базис тождеств третьей степени в $F_{2}^{W}$ составляет набор полиоднородных многочленов $F=\left\{f_{1}, \ldots, f_{n}\right\}$, то базис тождеств третьей степени в $F_{3}^{W}$ составляют линеаризачии тождеств из $F$ и, возмохнн, стандартное тохдество третьей степени.

ДОКАЗАТЕЛЬСТВО. Пусть

$$
f\left(x_{1}, x_{2}, x_{3}\right)=a_{0} x_{1} x_{2} x_{3}+a_{1} x_{2} x_{1} x_{3}+a_{2} x_{3} x_{1} x_{2}+a_{3} x_{3} x_{2} x_{1}+a_{4} x_{2} x_{3} x_{1}+a_{5} x_{2} x_{1} x_{3} .
$$

Если все следствия $f$ степени 3 от двух переменных тривиальны, то, в частности,

$f\left(x_{1}, x_{1}, x_{3}\right)=a_{0} x_{1} x_{1} x_{3}+a_{1} x_{1} x_{1} x_{3}+a_{2} x_{3} x_{1} x_{1}+a_{3} x_{3} x_{1} x_{1}+a_{4} x_{1} x_{3} x_{1}+a_{5} x_{1} x_{1} x_{3}=0$,

откуда $a_{1}+a_{0}=0, a_{2}+a_{3}=0$ и $a_{4}+a_{5}=0$. Аналогично, подставляя $x_{3}=x_{1}$, получаем $a_{0}=a_{2}=a_{4}=-a_{1}=-a_{3}=-a_{5}$, т.е. $f$ пропорционально St 3 . Таким образом, утверждение а) доказано. Из него следует утверждение б).

ПРЕДЛОЖЕНИЕ 1 (классификация многообразий). Пусть $W$ не совпадает ни одним из многообразий Ass, Com, Vect. Тогда многообразие $W$ попадает в один из следующих классов:

1) $m \geqslant 4$

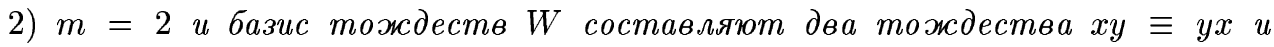
$x_{1} x_{2} \ldots x_{l} \equiv 0$ для некоторого $l \geqslant 3$

3) $m=2$ и базис тохдеств $W$ составляет тохдество $x y+y x \equiv 0$;

4) $m=3$ ив $F_{2}^{W}$ выполняется тохдество худ三0;

5) $m=3$ и ранг системы әлементов $\left\{x^{2} y, x y x, y x^{2}\right\}$ в $F_{2}^{W}$ равен 1 ( $x, y$ порохсдаюшие);

6) $m=3$ и ранг этой системы әлементов равен 3 , т.е. в $W$ из тожсдеств третьей степени выполняется только $\mathrm{St}_{3}$.

В остальных случаях $m=3$, ранг упомянутой системы әлементов в $F_{2}^{W}$ равен 2 и

7) в

8) из тохдеств степени 3 в $W$ выполняется только $x^{3} \equiv 0, и$ в $F_{2}^{W}$ есть еще тохудества степени 4 ;

9) из тохдеств степени 3 в $W$ выполняется только тождество $x^{3} \equiv 0, u$ все тохдества четвертой степени в $F_{2}^{W}$ следуют из него;

10) в $W$ выполняется $\mathrm{St}_{3}$, но не выполняется тождество $x^{3} \equiv 0$;

11) все тохдества третьей степени в $W$ - следствия тождества $\alpha x[x y]+$ $\beta[x y] x \equiv 0$ для некоторых $\alpha$ и $\beta$, не равных одновременно 0. 
ДокАЗАТЕЛЬСТво. Пусть $m=2$. Тогда в $W$ вьполняется некоторое тождество $f_{1}=a_{1} x y+a_{2} y x \equiv 0$, где $a_{1}$ и $a_{2}$ не равны одновременно 0 . Тогда в $W$ вьполняется и тождество $f_{2}=a_{1} y x+a_{2} x y \equiv 0$. Поскольку $W \neq$ Vect, система

$$
a_{1} x y+a_{2} y x=0, \quad a_{1} y x+a_{2} x y=0
$$

относительно $x y$ и $y x$ вырождена, т.е. $a_{1}^{2}-a_{2}^{2}=0$ и $a_{1}= \pm a_{2}$. Если $a_{1}=a_{2}$, то в $W$ выполняется тождество $x y+y x \equiv 0$. Из него следует тождество $x y z \equiv 0$ (поскольку $4 x y z \equiv 2[x, y z] \equiv[x[y z]] \equiv x y z-x z y-y z x+z y x \equiv-y x z+z x y+y x z-z x y \equiv 0)$, и больше никаких полилинейных тождеств наложить невозможно.

Если $a_{1}=-a_{2}$, то $f_{1}=a_{1}[x, y]$, и $[x y] \equiv 0$. Так как $W \neq$ Com, должно вьполняться еще какое-нибудь тождество большей степени, котороена многообразии коммутативньх алгебр сводится к мономиальному.

Пусть теперь $m=3$ и в $F_{2}^{W}$ не вьполняется тождество $x y z \equiv 0$. Тогда не все из элементов $\left\{x^{2} y, x y x, y x^{2}\right\}$ нулевые. Если ранг этой системы элементов равен 3 , то в $F_{2}^{W}$ никаких тождеств третьей степени не выполняется и по лемме 1 все тождества третьей степени порождаются стандартньм.

Пусть теперь ранг этой системы элементов равен 2 , т.е. в $F_{2}^{W}$ существует единственное соотношение, связьвающее эти элементы:

$$
f=a_{1} x^{2} y+a_{2} x y x+a_{3} y x^{2} \equiv 0 .
$$

Тогда согласно лемме 1 в $F_{3}^{W}$ из тождеств степени 3 может вьполняться, кроме следствий из $f$, только стандартное тождество.

Если в $W$ выполняется тождество $x^{3} \equiv 0$, то $f$ пропорционально его частичной линеаризации $x^{2} y+x y x+y x^{2} \equiv 0$. В противном случае при подстановке в выражение для $f$ значения $y=x$ получается тождественньй нуль, т.е. $a_{1}+a_{2}+a_{3}=0$, и $f=a_{1} x[x y]-a_{3}[x y] x$. Предложение доказано.

Лемма 2 (см. [2]). Пусть многообразие $W$ порохсдено тохсдеством $\alpha x[x y]+$ $\beta[x y] x \equiv 0$. Пусть $Q$ - пространство соотношений степени 4 и выше для относительно свободной алгебры $F_{n}^{W}, n \geqslant 1$.

Если $\alpha \neq 0, \beta \neq 0$ и $\alpha \pm \beta \neq 0$, то $Q$ порождено всевозможсными әлементами вида

$$
x_{1} \cdot \ldots \cdot x_{l}=x_{\sigma 1} \cdot \ldots \cdot x_{\sigma l}
$$

әде $\sigma \in S_{l}$, a $x_{i}-$ порохсдающие.

Если $\alpha \neq 0, \beta=0$, то пространство $Q$ порождено всевозможниыми әлементами вида

$$
x_{0} \cdot x_{1} \cdot \ldots \cdot x_{l}=x_{0} \cdot x_{\sigma 1} \cdot \ldots \cdot x_{\sigma l},
$$

а если $\alpha=0, \beta \neq 0$, то всевозможсными әлементами вида

$$
x_{1} \cdot \ldots \cdot x_{l} \cdot x_{0}=x_{\sigma 1} \cdot \ldots \cdot x_{\sigma l} \cdot x_{0} .
$$

В формулировке следующей леммы участвует понятие (некоммутативного) базиса Грёбнера; основные факты, связанные с ним, можно найти, например, в [3]. 
Лемма 3. Пусть $W$ - многообразие, порожденное тождеством $x^{3} \equiv 0, T-$ $T$-идеал соотношений алгебры $F_{2}^{W}$ с порождающими $x, y$ и пусть $F$ - редущированный базис Грёбнера идеала $T$ относительно лексикографически степенного порядка с $x>y$. Тогда все әлементы $F$ степени не выше 4 - это әлементы

$$
x^{3}, y^{3}, x y^{2}+y x y+y^{2} x, x^{2} y+x y x+y x^{2}, x y x y-y^{2} x^{2},
$$

а из мономов четвертой степени в $F_{2}^{W}$ нормальны только мономы

$$
x y x^{2}, y x y x, y^{2} x y, y^{2} x^{2}
$$

ДокАЗАТЕЛЬСТво. Идеал $T$-это $T$-идеал в $F_{2}^{\mathrm{Ass}}$, порожденньй элементом $x^{3}$. Чтобы вычислить элементы базиса Грёбнера степеней не выше 4, достаточно найти элементы этих степеней для какой-нибудь системы порождающих идеала. Такие элементы можно получить, подставляя в полную линеаризацию монома $x^{3}$, т.е. в многочлен $a b c+b a c+b c a+c b a+c a b+a c b$, вместо входящих в него переменных всевозможные мономы совокупной степени не вьше четвертой от переменных $x$ и $y$. Действуя таким образом, получаем следующую систему порождающих:

$$
\begin{gathered}
e_{1}=x^{3}, \quad e_{2}=y^{3}, \quad e_{3}=y x^{2}+x y x+x^{2} y, \quad e_{4}=y^{2} x+y x y+x y^{2}, \\
e_{5}=2 y x y x+y^{2} x^{2}+y x^{2} y+x y x y+x y^{2} x, \quad e_{6}=x y^{2} x+y x y x+2 x y x y+x^{2} y^{2}+y x^{2} y, \\
e_{7}=x^{2} y x+2 y x^{3}+2 x^{3} y+x y x^{2}, \quad e_{8}=2 y^{3} x+y^{2} x y+2 x y^{3}+y x y^{2}
\end{gathered}
$$

Поскольку все мономы, входящие в разложения порождающих третьей степени, различны, то $e_{1}, e_{2}, e_{3}, e_{4}$ принадлежат редуцированному базису Грёбнера. Элементы $e_{7}$ и $e_{8}$ редуцируются относительно них к нулю и в построении базиса Грёбнера не участвуют, равно как и все три $s$-формы $x e_{4}-e_{3} y, e_{1} y-x e_{3}$ и $x e_{2}-e_{4} y$, имеющие степень не вьше 4. Оба оставшихся элемента $e_{5}$ и $e_{6}$ редуцируются к элементу $x y x y-y^{2} x^{2}$, который, таким образом, тоже входит в базис Грёбнера, и больше элементов третьей и четвертой степени в нем нет.

Теперь нормальньми будут в точности те мономы четвертой степени, которые не делятся на старшие члены элементов базиса Грёбнера степеней не выше 4, что и требовалось.

При фиксированном $W$ через $A_{C}$ будем обозначать алгебру $A \stackrel{W}{*} F_{1}^{W}$, где $A \in \mathrm{Ob} W$; порождающую подалгебры $F_{1}^{W} \subset A_{C}$ будем обозначать буквой $c$, считая $\operatorname{deg} c=1$.

Лемма 4. Пусть все соотношения алгебры А полиоднородны по порождающим. Тогда алгебры $A_{C}, \bar{A}$ и $\bar{A}_{C}=(\bar{A})_{C}$ полиградуированы по степеням всех порожсдаюuция.

ДокАЗАТЕЛЬСТво. Все соотношения алгебры $A_{C}$ мономиальны, поэтому она полиградуирована. Поскольку для всякой полиградуированной алгебры $D$ в алгебре $D_{C}$ к ее соотношениям добавляются только тождества из $W$, которые порождаются (как линейное пространство) полиоднородными тождествами, то $D_{C}$ полиградуирована. Осталось положить $D=A$ и $D=\bar{A}$.

2. Основная теорема. Основной результат данной работы составляет следующая 
Tеорема. Во всяком многообразии $W$, отличном от Ass, Com, Vect, существуют две градуированные алгебры $A$ и $B$ такие, что $A(t)=B(t)$, но $A_{C}(t) \neq$ $B_{C}(t)$.

ДоказАТЕльСтво. Для $D \in \mathrm{Ob} W$ через $T_{D} \triangleleft D \stackrel{\text { Ass }}{*} F_{1}^{W}$ обозначим идеал такой, что $D_{C}=\left(D^{\text {Ass }} * F_{1}^{W}\right) / T_{D}$.

Мы используем ту же нумерацию классов многообразий, что и в формулировке предложения 1.

1) Пусть $m \geqslant 4$. Положим $X=\left\{a_{1}, \ldots, a_{m-1}\right\}, \operatorname{deg} a_{1}=\cdots=\operatorname{deg} a_{m-1}=1$, $A=k\left\langle X \mid x_{1} \cdots x_{m}=0, x_{i} \in X\right\rangle, B=\bar{A}$. Будем доказьвать, что $\operatorname{dim}\left(A_{C}\right)_{m} \neq$ $\operatorname{dim}\left(B_{C}\right)_{m}$. Для этого достаточно убедиться, что $\operatorname{dim}\left(T_{A}\right)_{m} \neq \operatorname{dim}\left(T_{B}\right)_{m}$.

Пусть $F=F^{W}(X)$ и пусть $P, P_{A}, P_{B}$ - подпространства полилинейных многочленов от порождающих первой степени в $\left(T_{F}\right)_{m},\left(T_{A}\right)_{m}$ и $\left(T_{B}\right)_{m}$ соответственно. Из минимальности $m$ следует, что естественные гомоморфизмы векторных пространств $\varphi$ : $\left(T_{F}\right)_{m} \rightarrow\left(T_{A}\right)_{m}$ и $\psi:\left(T_{F}\right)_{m} \rightarrow\left(T_{B}\right)_{m}$ сюрьективны, причем $\varphi(P) \subset P_{A}, \psi(P) \subset P_{B}$.

Пусть $\left(T_{F}\right)_{m}=T_{1} \oplus T_{2}$, где $T_{1}$ - подпространство многочленов, не зависящих от $c$. Так как $\varphi T_{1}=0$ и $\psi T_{1}=0$, a $\left.\varphi\right|_{T_{2}}$ изоморфизм (поскольку в размерностях меньших $m$ $A$ изоморфно $F)$, достаточно доказать, что $\operatorname{ker} \psi \cap P \neq 0$.

Гомоморфизм $\psi_{P}=\left.\psi\right|_{P}: P \rightarrow B_{C}$ отображает многочлен

$$
f=\sum_{\sigma \in S_{m}} \alpha_{\sigma} a_{\sigma 1} \cdots a_{\sigma m}
$$

в многочлен

$$
\psi_{P}(f)=\sum_{\sigma \in S_{m}} \alpha_{\sigma} \bar{a}_{\sigma 1} \cdots \bar{a}_{\sigma m}
$$

где $a_{m}=c, \bar{a}_{m}=c$.

Поскольку $m \geqslant 4$, то в разложении $\psi_{P}(f)$ в каждом одночлене встретится произведение вида $\bar{a}_{i} \bar{a}_{j}$, где $i, j<m$, и $\psi_{P}(f)=0$ (так как умножение в $B$ нулевое). Таким образом, $P=\operatorname{ker} \psi \cap P \neq 0$, что и требовалось.

2) Положим $A=k\left\langle a \mid a^{l}=0\right\rangle, B=\bar{A}$. Тогда в $A_{C}$ нет элементов степени $l$, а в $B_{C}$ есть элемент $\overline{a^{l-1}} \cdot c$, откуда $0=\operatorname{dim}\left(A_{C}\right)_{l}<\operatorname{dim}\left(B_{C}\right)_{l}$.

$3)$ В этом случае положим $A=F_{2}^{W}, B=\bar{A}$. Тогда $\left(A_{C}\right)_{3}=0$, ав $\left(B_{C}\right)_{3}$ есть элемент $\overline{a b} \cdot c$.

4), 5) В этих случаях ранг системы элементов $\left\{x^{2} y, x y x, y x^{2}\right\} \subset F_{2}^{W}$ не больше 1. Поэтому $A=k\left\langle a \mid a^{3}=0\right\rangle, B=\bar{A}$.

По лемме 4 пространства $\left(A_{C}\right)_{3}$ и $\left(B_{C}\right)_{3}$ градуированы по степеням $c$ и $\left(A_{C}\right)_{3}=A_{3} \oplus$ $A^{\prime} \oplus A^{\prime \prime},\left(B_{C}\right)_{3}=B_{3} \oplus B^{\prime} \oplus B^{\prime \prime}$, где

$$
\begin{array}{ll}
A^{\prime}=k\left\{c^{3}, c a c, a c^{2}, c^{2} a\right\}, & A^{\prime \prime}=k\left\{a^{2} c, c a^{2}, a c a\right\}, \\
B^{\prime}=k\left\{c^{3}, c \bar{a} c, \bar{a} c^{2}, c^{2} \bar{a}\right\}, & B^{\prime \prime}=k\left\{\overline{a^{2}} c, c \overline{a^{2}}, \bar{a} c \bar{a}\right\} .
\end{array}
$$

Очевидно, $\operatorname{dim} A_{3}=\operatorname{dim} B_{3}, \operatorname{dim} A^{\prime}=\operatorname{dim} B^{\prime}$. При этом $\operatorname{dim} A^{\prime \prime} \leqslant 1$, а в $B^{\prime \prime}$ содержатся два линейно независимых элемента $\overline{a^{2}} c$ и $c \bar{a}^{2}$, откуда $\operatorname{dim} B^{\prime \prime} \geqslant 2$ и $\operatorname{dim}\left(A_{C}\right)_{3}<$ $\operatorname{dim}\left(B_{C}\right)_{3}$. 
6) В этом случае в $W$ выполняется единственное тождество третьей степени

$$
s(x, y, z)=x[y, z]+y[z, x]+z[x, y] \equiv 0 .
$$

Пусть $A^{1}=k\left\langle a \mid a^{3}=0\right\rangle, B^{1}=\bar{A}^{1}$. Если $\operatorname{dim}\left(A_{C}^{1}\right)_{4} \neq \operatorname{dim}\left(B_{C}^{1}\right)_{4}$, то эти алгебры и составляют искомьй пример. Предположим, что $\operatorname{dim}\left(A_{C}^{1}\right)_{4}=\operatorname{dim}\left(B_{C}^{1}\right)_{4}$.

Пусть $A=k\left\langle a, b \mid a^{3}=b^{3}=a b=b a=0\right\rangle, B=\bar{A}$. Докажем, что $\operatorname{dim}\left(A_{C}\right)_{4} \neq \operatorname{dim}\left(B_{C}\right)_{4}$.

Заметим, что подалгебры $L^{1}$ и $L^{2}$ в $A_{C}$, порожденные множествами $\{a, c\}$ и $\{b, c\}$, изоморфны $A_{C}^{1}$, а их пересечение изоморфно $F_{1}^{W}$; аналогично, подалгебры $M^{1}$ и $M^{2}$ в $B_{C}$, порожденные множествами $\left\{\bar{a}, \overline{a^{2}}, c\right\}$ и $\left\{\frac{\bar{b}}{b^{2}}, c\right\}$, изоморфны $B_{C}^{1}$, а их пересечение изоморфно $F_{1}^{W}$. Таким образом, нам достаточно сравнивать размерности прямых дополнений к $\left(L^{1} \oplus L^{2}\right)_{4}$ в $\left(A_{C}\right)_{4}$ и к $\left(M^{1} \oplus M^{2}\right)_{4}$ в $\left(B_{C}\right)_{4}$. Зафиксируем такие дополнения - пространства $R$ и $S$. Они порождены мономами четвертой степени из $\left(A_{C}\right)_{4}$ и $\left(B_{C}\right)_{4}$ соответственно, в запись которых входят все три буквы $a, b, c$.

Для десяти мономов из $R$ вьполняются следуюшие девять соотношений:

$$
\begin{gathered}
s\left(a, b, c^{2}\right)=-a c^{2} b+b c^{2} a=0, \quad s(a, c, b c)=a c b c+c b c a+b c a c-b c^{2} a=0, \\
s(b, c, a c)=b c a c+c a c b+a c b c-a c^{2} b=0, \quad s(a, c, c b)=a c^{2} b-a c b c-c a c b-c b c a=0, \\
s(b, c, c a)=b c^{2} a-b c a c-c b c a-c a c b=0, \quad s(b, a, a c)=a^{2} c b=0, \\
s(a, b, b c)=b^{2} c a=0, \quad s(a, b, c a)=b c a^{2}=0, \quad s(b, a, c b)=a c b^{2}=0,
\end{gathered}
$$

откуда

$$
b c^{2} a=a c^{2} b=a c b c=b c a c=c a c b=c b c a,
$$

и вне зависимости от остальных тождеств $W \operatorname{dim} R \leqslant 1$.

С другой стороны, в $S$ входят мономы $\overline{a^{2}} c \bar{b}, \bar{b} c \overline{a^{2}}, \bar{a} c \bar{b}^{2}$ и $\overline{b^{2}} c \bar{a}$, которые связаны только соотношениями

$$
s\left(\overline{a^{2}}, c, \bar{b}\right)=\overline{a^{2}} c \bar{b}-\bar{b} c \overline{a^{2}}, \quad s\left(\overline{b^{2}}, c, \bar{a}\right)=\overline{b^{2}} c \bar{a}-\bar{a} c \overline{b^{2}} .
$$

Таким образом, $\operatorname{dim} S \geqslant 2>\operatorname{dim} R$ и $\operatorname{dim}\left(A_{C}\right)_{4}>\operatorname{dim}\left(B_{C}\right)_{4}$.

7) В этом случае все тождества степени 3 в $F_{2}^{W}$ порождены тождествами $x^{3} \equiv 0$ и $\mathrm{St}_{3}$.

Положим $A=k\left\langle a \mid a^{3}=0\right\rangle=F_{1}^{W}, B=\bar{A}$. Докажем сначала, что алгебра $A_{C}=$ $F_{2}^{W}$ нильпотентна степени 4 . Поскольку тождество $x^{3} \equiv 0$ выполняется в $W$, то каждьй однородньй элемент четвертой степени в $F_{2}^{W}$ представим в виде линейной комбинации нормальных мономов из леммы 3 . Таким образом, достаточно доказать, что в $F_{2}^{W}$ с порождающими $x, y$ мономы $x y x^{2}, y x y x, y^{2} x y, y^{2} x^{2}$ равны 0 .

Действительно, имеем $s\left(x, y, x^{2}\right)=x y x^{2}-x^{2} y x \equiv 0$ и из линеаризации тождества $x^{3} \equiv 0$

$$
x^{2} y x+x y x^{2} \equiv x\left(x^{2} y+x y x+y x^{2}\right) \equiv 0,
$$

откуда $x y x^{2} \equiv x^{2} y x \equiv 0$, и после замены переменных получаем $y^{2} x y \equiv 0$.

Кроме того,

$$
s(y, x, y x)=x y x y-y^{2} x^{2}-x y^{2} x+2 y x y x-y x^{2} y \equiv 0 .
$$


Редуцируя среднюю часть последнего равенства с помощью элементов базиса Грёбнеpa, из леммы 3 получаем $2 y x y x+y^{2} x^{2} \equiv 0$. Аналогично, из тождества $s(y, x, y x) \equiv 0$ получаем равенство - $y x y x+2 y^{2} x^{2} \equiv 0$, откуда $y x y x=y^{2} x^{2}=0$.

Теперь достаточно заметить, что $0 \neq c^{2} \overline{a^{2}} \in\left(B_{C}\right)_{4}$ и $0=\operatorname{dim}\left(A_{C}\right)_{4}<\operatorname{dim}\left(B_{C}\right)_{4}$.

8) В этом случае в $W$ из тождеств третьей степени выполняется только $x^{3} \equiv 0$ и в $F_{2}^{W}$ выполняется еще какое-то тождество четвертой степени $f \equiv 0$.

Как и прежде, положим $A=k\left\langle a \mid a^{3}=0\right\rangle=F_{1}^{W}, B=\bar{A}$. Тогда $A_{C}=F_{2}^{W}$.

Можно считать, что $f$ полиоднородно по переменным; кроме того, по лемме 3 можно считать, что $f$ является линейной комбинацией мономов от порождающих $x y x^{2}, y x y x$, $y^{2} x y, y^{2} x^{2}$.

Если $f$ имеет степень 3 по одной переменной и степень 1 по другой, то $x y x^{2} \equiv 0$ и $y^{2} x y \equiv 0$, откуда $\operatorname{dim}\left(A_{C}\right)_{4} \leqslant 2$, и тогда, поскольку элементы $\overline{a^{2}} c^{2}, c^{2} \overline{a^{2}}$ и $\bar{a} c \overline{a^{2}}$ из $B_{C}$ линейно независимы, $\operatorname{dim}\left(B_{C}\right)_{4} \geqslant 3>\operatorname{dim}\left(A_{C}\right)_{4}$.

Если же все тождества степени 4 в $F_{2}^{W}$, кроме следствий из тождества $x^{3} \equiv 0$, имеют степень 2 по обеим переменным, то элемент $c^{2} \bar{a} c$ линейно не зависит от перечисленных выше, откуда $\operatorname{dim}\left(B_{C}\right)_{4} \geqslant 4>\operatorname{dim}\left(A_{C}\right)_{4}$.

9) В этом случае в $W$ вьполняется тождество $x^{3} \equiv 0$, и все тождества степеней 3 и 4 в $F_{2}^{W}$ являются его следствиями.

Положим $A=k\left\langle a, b \mid a^{2}=b^{2}=a b=0\right\rangle, B=\bar{A}=k\{a, b, q \mid \operatorname{deg} a=\operatorname{deg} b=1$, $\operatorname{deg} q=2\}$. Докажем, что $\operatorname{dim}\left(B_{C}\right)_{4} \geqslant 6>\operatorname{dim}\left(A_{C}\right)_{4}$.

Первая часть неравенства следует из того, что в алгебре $B$ есть шесть линейно независимых элементов $c^{2} q, q c^{2}, a c q, b c q, c^{2} a c, c^{2} b c$ (независимость последних двух элементов следует из леммы 3 ).

Вторую часть неравенства достаточно доказать для случая, когда $W$ порождается тождеством $x^{3} \equiv 0$, т.е. все тождества следуют из его линеаризации

$$
u\left(x_{1}, x_{2}, x_{3}\right)=\sum_{\sigma \in S_{3}} x_{\sigma 1} x_{\sigma 2} x_{\sigma 3} \equiv 0 .
$$

По лемме 3 пространство полиоднородных элементов четвертой степени в $\left(A_{C}\right)_{4}$, зависяших только от двух переменньх, порождается двумя элементами $c a c^{2}$ и $c b c^{2}$. Из тождеств $u(x, a, a) \equiv 0$ и $u(x, b, b) \equiv 0$, где $x$ - любой элемент, получаем соотношения $a x a=0$ и $b x b=0$; поэтому все ненулевые слова могут включать буквы $a$ и $b$ не более чем по одному разу. Значит, все ненулевые полиоднородные элементы четвертой степени, зависящие от всех трех переменных, имеют степень 2 по $c$ и степень 1 по $a$ и по $b$; обозначим пространство таких элементов через $P$.

Из тождеств $u(c, c, b) \equiv 0, u(c, c, a) \equiv 0, u(c, b a, c) \equiv 0$ следуют соотношения

$$
c^{2} a+c a c+a c^{2}=0, \quad c^{2} b+c b c+b c^{2}=0, \quad c^{2} b a+c b a c+b a c^{2}=0 .
$$

Поэтому все мономы из $P$ представимы в виде линейных комбинаций мономов, делящихся на $c^{2}$. Среди таких мономов не более четырех ненулевьх (т.е. не делящихся на $a b$ ), и они связаны соотношением $u\left(a, b, c^{2}\right)=0$. Таким образом, $\operatorname{dim} P \leqslant 3$ и $\operatorname{dim}\left(A_{C}\right)_{4} \leqslant 5$.

$10), 11)$ Все тождества степени 3 в $W$ - следствия тождества

$$
f(x, y)=a_{1} x[x, y]+a_{2}[x, y] x \equiv 0
$$

и, возможно, $\mathrm{St}_{3}$. 
Предположим сначала, что $a_{1}+a_{2}=0$, т.е. все тождества третьей степени в $F_{2}^{W}-$ следствия тождества $x^{2} y-y x^{2} \equiv 0$. Пусть тогда $A=k\left\langle a \mid a^{3}=0\right\rangle, B=\bar{A}$. Как и при рассмотрении случаев 4$)$ и 5$)$ выше, разложим $\left(A_{C}\right)_{3}$ и $\left(B_{C}\right)_{3}$ в прямые суммы:

$$
\left(A_{C}\right)_{3}=A_{3} \oplus A^{\prime} \oplus A^{\prime \prime}, \quad\left(B_{C}\right)_{3}=B_{3} \oplus B^{\prime} \oplus B^{\prime \prime} .
$$

В этом случае по-прежнему $\operatorname{dim} A_{3}=\operatorname{dim} B_{3}, \operatorname{dim} A^{\prime}=\operatorname{dim} B^{\prime}$, но пространство $A^{\prime \prime}$ порождено двумя линейно независимьми элементами аса и $\mathrm{ca}^{2}$, а пространство $B^{\prime \prime}$ - тремя линейно независимыми элементами $\bar{a} c \bar{a}, \overline{a^{2}} c$ и $c \overline{a^{2}}$. Значит, $\operatorname{dim}\left(A_{C}\right)_{3}<\operatorname{dim}\left(B_{C}\right)_{3}$.

Рассмотрим теперь случай, когда $a_{1} \pm a_{2} \neq 0, a_{1} \neq 0$ и $a_{2} \neq 0$. По-прежнему положим $A=k\left\langle a \mid a^{3}=0\right\rangle, B=\bar{A}$. По лемме 2 пространство $\left(A_{C}\right)_{4}$ порождается элементами $a^{2} c^{2}, a c^{3}, c^{4}$ и $\operatorname{dim}\left(A_{C}\right)_{4} \leqslant 3$. С другой стороны, алгебра $B_{C}$ содержит линейно независимые элементы $\overline{a^{2}} c^{2}, c^{2} \overline{a^{2}}, \bar{a} c^{3}, c^{4}$, откуда $\operatorname{dim}\left(A_{C}\right)_{4}<4 \leqslant \operatorname{dim}\left(B_{C}\right)_{4}$.

Пусть теперь $a_{1}-a_{2}=0$, т.е. $f$ сводится к тождеству $[x[x y]] \equiv 0$, которое, в свою очередь, сводится к тождеству $[x[y z]] \equiv 0$ (см. [2]). Положим $A=k\langle a, b| x y z=0, x, y, z \in$ $\{a, b\},[a, b]=0\rangle, B=k\langle a, b \mid x y z=0, x, y, z \in\{a, b\}, a b=0\rangle$. Тогда пространства $\left(T_{A}\right)_{3}$ и $\left(T_{B}\right)_{3}$ порождены элементами $[[a c] c],[[b c] c],[[a c] a],[[b c] b],[[a b] c],[[c a] b]$ (третий полилинейньй длинньй коммутатор выражается через два других по тождеству Якоби) и, если выполняется $\mathrm{St}_{3}$ (случай 10)), возможно, элементом $s(a, b, c)=a[b c]+$ $c[a b]+b[c a]$. При этом в идеале $T_{A}$ вьполняется равенство $[a, b]=0$, откуда $[[a b] c]=0$.

Если $\mathrm{St}_{3}$ не вьполняется (случай 11$)$ ), то в идеале $T_{B}$ все шесть перечисленных длинных коммутаторов линейно независимы, и $\operatorname{dim}\left(T_{A}\right)_{3}=5<6=\operatorname{dim}\left(T_{B}\right)_{3}$, откуда $\operatorname{dim}\left(A_{C}\right)_{3} \geqslant \operatorname{dim}\left(B_{C}\right)_{3}$. Если же выполняется еще $\mathrm{St}_{3}$, то пространства полилинейных элементов $P_{A}$ и $P_{B}$ в $T_{A}$ и $T_{B}$ соответственно порождены элементами $s(a, b, c),[[a b] c]$, $[[c a] b]$. Очевидно, $\operatorname{dim} P_{A} \leqslant 2$ и все три порождающих пространства $P_{B}$

$s(a, b, c)=-a c b-c b a+b c a-b a c, \quad[[a b] c]=-b a c+c b a, \quad[[c a] b]=-a c b-b c a+b a c$

линейно независимы и, следовательно, $\operatorname{dim}\left(A_{C}\right)_{3} \geqslant \operatorname{dim}\left(B_{C}\right)_{3}$.

Рассмотрим теперь случай, когда $a_{2}=0$ (случай $a_{1}=0$ совершенно аналогичен). Тогда все тождества третьей степени в $F_{2}^{W}$ порождаются тождеством $x[x, y] \equiv 0$. Пусть $A=F_{1}^{W}, B=\bar{A}$. Тогда $A_{C}=F_{2}^{W}$, и по лемме 2 пространство $\left(A_{C}\right)_{4}$ порождено восемью элементами $c^{4}, a c^{3}, c^{3} a, a^{2} c^{2}, c^{2} a^{2}, a^{3} c, c a^{3}, a^{4}$, а пространство $\left(B_{C}\right)_{4}$ - девятью элементами $c^{4}, \bar{a} c^{3}, c^{3} \bar{a}, \overline{a^{2}} c^{2}, c^{2} \overline{a^{2}}, \overline{a^{3}} c, c \bar{a}^{3}, \overline{a^{4}}, \overline{a^{2}} c \bar{a}$. Если все тождества четвертой степени от двух переменных являются следствиями тождества $x[x, y] \equiv 0$, то все перечисленные элементы линейно независимы и $\operatorname{dim}\left(A_{C}\right)_{4}=8<9=\operatorname{dim}\left(B_{C}\right)_{4}$.

Предположим, что в $F_{2}^{W}$ вьполняется еще какое-то тождество четвертой степени. Заметим, что из перечисленных порождающих пространства $\left(B_{C}\right)_{4}$ только элементы $c^{4}, c^{3} \bar{a}, \bar{a} c^{3}$ имеют степень 4 по переменным, и поэтому остальные порождающие линейно независимы. С другой стороны, ранг $r$ системы элементов $\left\{c^{4}, c^{3} \bar{a}, \bar{a} c^{3}\right\}$ в $B$ равен рангу системы элементов $\left\{c^{4}, a c^{3}, c^{3} a\right\}$ в $A$ (это следует из леммы 4$)$, откуда $\operatorname{dim}\left(A_{C}\right)_{4} \leqslant 5+$ $r<6+r=\operatorname{dim}\left(B_{C}\right)_{4}$.

Теорема доказана полностью.

3. Стандартные алгебры. Можно несколько сузить исходную постановку задачи, рассматривая только стандартные алгебры, т.е. градуированные алгебры, порожденные своими элементами первой степени. Как показьвает рассмотренньй выше (в 
рамках случаев 10) и 11)) случай многообразия $W$, в котором все тождества степени не выше третьей следуют из тождества $x[x y] \equiv 0$, даже в классе стандартных алгебр ряд Гильберта алгебры $A_{C}$ не определяется рядом Гильберта алгебры $A$. Аналогичные примеры можно привести и для других многообразий, например, для многообразия, порожденного тождеством $x^{3} \equiv 0$. Тем не менее, существуют по крайней мере две бесконечных возрастающих цепочки многообразий, в которых ряд Гильберта свободного произведения определяется рядами Гильберта сомножителей.

Пусть многообразие $W_{i}, i \geqslant 2$, порождается тождеством $x_{1} \cdots x_{i} \equiv 0$, и многообразие $W^{l}, l \geqslant 2$, порождается всеми полилинейными тождествами многообразия Com, имеющими степень не меньше $l$.

ПРЕДЛОЖЕНИЕ 2. Пусть $W$ - одно из многообразий $W_{i}, W^{l}$ и пусть $A$ и $B$-две стандартные алгебры из $W$. Тогда, зная ряды Гильберта алгебр $A$ и $B$, можно определить ряды Гильберта алгебры $A \stackrel{W}{*} B$.

ДокАЗАТЕЛЬСТво. Пусть $A(t)=a_{1} t+a_{2} t^{2}+\cdots$ и $B(t)=b_{1} t+b_{2} t^{2}+\cdots$. Обозначим

$$
\begin{array}{ll}
C=A \stackrel{W}{*} B, & C(t)=c_{1} t+c_{2} t^{2}+\cdots, \\
P=A \stackrel{*}{\text { Com }} B, & P(t)=(A(t)+1)(B(t)+1)-1=p_{1} t+p_{2} t^{2}+\cdots, \\
Q=A \stackrel{\text { Ass }}{*} B, & Q(t)=\frac{1}{(A(t)+1)^{-1}+(B(t)+1)^{-1}-1}-1=q_{1} t+q_{2} t^{2}+\cdots .
\end{array}
$$

Если $W=W_{i}$, то до $(i-1)$-й степени алгебра $C$ как линейное пространство изоморфна $Q$, а начиная с $i$-й степени все компоненты нулевые, поэтому

$$
C(t)=q_{1} t+\cdots+q_{i-1} t^{i-1}
$$

Если же $W=W^{l}$, то до $(l-1)$-й степени алгебра $C$ как линейное пространство изоморфна $Q$, а начиная с $l$-й степени изоморфна $P$, поэтому

$$
C(t)=q_{1} t+\cdots+q_{l-1} t^{l-1}+p_{l} t^{l}+p_{l+1} t^{l+1}+\cdots
$$

\section{СПИСОК ЦИТИРОВАННОЙ ЛИТЕРАТУРЫ}

[1] Белов А.Я. О рациональности рядов Гильберта относительно свободных алгебр // УМН. 1997. T. 52. № 2. C. 153-154.

[2] Ананьин А. З., Кемер А.Р. Многообразия ассоциативных алгебр, решетки подмногообразий которых дистрибутивны // Сиб. матем. ж. 1976. Т. 17. № 4. С. 723-730.

[3] Уфнаровский В. А. Комбинаторные и асимптотические методы в алгебре // Современная матем. и ее прилож. 1990. Т. 57. С. 5-177.

Московский государственный университет им. М.В. Ломоносова

Поступило

E-mail: piont@mech.math.msu.su 